\title{
Níveis de fósforo total em dietas para alevinos de tilápia-do-nilo
}

\author{
Felipe Barbosa Ribeiro1, Eduardo Arruda Teixeira Lanna², Marcos Antonio Delmondes Bomfim ${ }^{3}$, \\ Juarez Lopes Donzele ${ }^{2}$, Anderson Saraiva de Freitas ${ }^{4}$, Maíra Paula de Sousa ${ }^{4}$, Moisés Quadros ${ }^{4}$

\footnotetext{
2 Departamento de Zootecnia DZO/UFV - Viçosa-MG

${ }^{3}$ Doutorando em Zootecnia - UFV - Viçosa-MG.
} \\ ${ }^{1}$ Mestre em Zootecnia DZO/UFV - Viçosa-MG. \\ ${ }^{4}$ Mestrando em Zootecnia UFV - Viçosa-MG.
}

RESUMO - Objetivando-se determinar a exigência de fósforo em dietas para alevinos de tilápia-do-nilo (Oreochromis niloticus), foram utilizados 432 alevinos com peso inicial de $0,60 \pm 0,02 \mathrm{~g}$, mantidos em 36 aquários de 150 L. Foi utilizado delineamento inteiramente casualizado, com seis tratamentos $(0,55 ; 0,73 ; 0,94 ; 1,14 ; 1,37$ e $1,59 \%$ de fósforo total), seis repetições e 12 peixes por unidade experimental. Os peixes foram alimentados com rações contendo $32 \%$ de PB e $3.000 \mathrm{kcal}$ $\mathrm{ED} / \mathrm{kg}$, durante 40 dias. Verificou-se efeito quadrático dos níveis de fósforo na dieta sobre a conversão alimentar e a taxa de eficiência protéica, sendo estimados os melhores resultados com 1,10\% de fósforo total. Houve efeito linear dos níveis de fósforo na dieta sobre a taxa de eficiência de retenção de $\mathrm{P}$ e a taxa de eficiência de $\mathrm{P}$. Para as demais variáveis, não houve efeito dos níveis de fósforo da dieta. A exigência de $\mathrm{P}$ em dietas para alevinos de tilápia-do-nilo é de $1,10 \%$.

Palavras-chave: alevinos, fósforo total, Oreochromis niloticus

\section{Dietary total phosphorus levels for Nile tilapia fingerlings}

\begin{abstract}
Four hundred and thirty-two Nile tilapia (Oreochromis niloticus) fingerlings averaging initial weight of $0.60 \pm 0.02 \mathrm{~g}$ were allotted to thirty-six $150 \mathrm{~L}$-aquaria to determine total phosphorus requirement. The experiment was analyzed as a completely randomized design, with six treatments $(0.55,0.73,0.94,1.14,1.37$, and $1.59 \%$ of total phosphorus), six replicates and 12 fishes per experimental unit. The fishes were fed diets with $32 \%$ of CP and 3,000 kcal of DE $/ \mathrm{kg}$, during 40 days. Quadratic effect was observed for feed/gain ratio and protein efficiency rate and the best results were estimated with $1.10 \%$ of total phosphorus, for both variables. Linear effect of the dietary phosphorus levels on $\mathrm{P}$ retention efficiency rate and $\mathrm{P}$ efficiency rate was noticed. No treatment effect on the other variables was observed. The dietary total phosphorus requirement for Nile tilapia corresponds to $1.10 \%$.
\end{abstract}

Key Words: fingerlings, total phosphorus, Oreochromis niloticus

\section{Introdução}

Entre as espécies de peixes de interesses econômico e social, destaca-se a tilápia-do-nilo(Oreochromis niloticus), uma espécie criada principalmente nos países tropicais e subtropicais, em virtude de seu rápido crescimento em sistema intensivo, da facilidade de obtenção de larvas, da rusticidade e do hábito alimentar onívoro. Além disso, sua carne possui boas características organolépticas e seu filé não possui espinhos intramusculares em forma de "Y" (Hildsorf, 1995), o que facilita a industrialização para comercialização nos mercados interno e externo.

Apesar de todas essas características, são poucas as informações acerca das exigências de minerais para tilápiado-nilo que permitem a formulação de dietas balanceadas. Adicionalmente, o número reduzido de pesquisas no Brasil força a adoção de recomendações obtidas em outros países, que, muitas vezes, não são aplicáveis às nossas condições.

Entre os minerais essenciais, o cálcio e o fósforo tem sido apontados como aqueles exigidos em altos níveis pelos animais em comparação aos demais minerais. Tem-se observado que, em peixes, 65,0 a $80,0 \%$ das exigências de cálcio podem ser supridas diretamente da água, por meio de absorção ativa pelas brânquias, enquanto o fósforo deve ser oferecido na dieta (Simkiss, 1974; Wilson et al., 1982).

O fósforo é considerado um nutriente essencial para formação da estrutura óssea e o metabolismo corporal, sendo imprescindível que esteja em nível adequado nas rações para atender à exigência nutricional do animal. Além disso, esse mineral, em concentrações excessivas no meio aquático pode levar a eutrofização do meio, comprometendo a qualidade da água e a capacidade de suporte dos sistemas 
aqüícolas (Van Der Ploeg \& Boyd, 1991; English et al., 1993) e, no caso de predominância de cianobactérias, prejudicar as características organolépticas da carcaça dos peixes (Van Der Ploeg \& Tucker, 1994). Portanto, torna-se necessário que o nível de fósforo na ração atenda estritamente à exigência do animal.

Assim, conduziu-se um experimento para determinar a exigência de fósforo total para alevinos de tilápia-do-nilo (Oreochromis niloticus) utilizando rações práticas.

\section{Material e Métodos}

O experimento foi conduzido no período de 14 de dezembro de 2004 a 24 de janeiro de 2005, no Laboratório de Nutrição de Peixes do Departamento de Zootecnia da Universidade Federal de Viçosa (UFV), em Viçosa, MG.

Foram utilizados 432 alevinos revertidos de tilápia (Oreochromis niloticus), da linhagem tailandesa, com peso inicial de 0,60 $\pm 0,02 \mathrm{~g}$, obtidos de mesma desova, distribuídos em delineamento inteiramente casualizado, com seis tratamentos, seis repetições e 12 peixes por unidade experimental.

Os tratamentos foram constituídos de uma dieta basal, isoenergética e isoprotéica, formulada para atender às exigências nutricionais mínimas sugeridas pelo NRC (1993), com exceção do fósforo. A dieta basal foi suplementada com seis níveis de fosfato monoamônio, resultando em seis dietas experimentais com 0,$50 ; 0,70 ; 0,90 ; 1,10 ; 1,30$ e $1,50 \%$ de fósforo total (Tabela 1 ).

Foram utilizados 36 aquários de polietileno, com capacidade de $150 \mathrm{~L}$, dotados de sistemas individuais de abastecimento de água, aeração e escoamento de fundo.

A água de abastecimento dos aquários, proveniente do sistema de tratamento de água da Universidade Federal de Viçosa - UFV, foi previamente declorada e aquecida por resistências elétricas, com temperatura controlada por termostato, sendo mantida a $28^{\circ} \mathrm{C}$.

A temperatura da água foi aferida diariamente, às $8 \mathrm{e}$ 17h, com o auxílio de um termômetro de bulbo de mercúrio, graduado de 0 a $50^{\circ} \mathrm{C}$. Os controles do $\mathrm{pH}$ e do teor de

Tabela 1 - Composição das dietas experimentais (matéria natural)

Table 1 - Ingredient and chemical compositions of the experimental diets (on as-fed)

\begin{tabular}{|c|c|c|c|c|c|c|}
\hline \multirow[t]{2}{*}{$\begin{array}{l}\text { Item }(\%) \\
\text { Item }(\%)\end{array}$} & \multicolumn{6}{|c|}{$\begin{array}{l}\text { Nível de fósforo total (\%) } \\
\text { Total phosphorus level (\%) }\end{array}$} \\
\hline & 0,50 & 0,70 & 0,90 & 1,10 & 1,30 & 1,50 \\
\hline Farelo de soja $(45 \%)$ (Soybean meal) & 66,30 & 66,30 & 66,30 & 66,30 & 66,30 & 66,30 \\
\hline Fubá de milho $(8,57 \%)$ (Corn meal) & 20,67 & 20,67 & 20,67 & 20,67 & 20,67 & 20,67 \\
\hline Óleo de soja (Soybean oil) & 5,10 & 5,10 & 5,10 & 5,10 & 5,10 & 5,10 \\
\hline Inerte (areia) (Inert, sand) & 5,00 & 4,16 & 3,33 & 2,49 & 1,66 & 0,82 \\
\hline Fosfato monoamônio (Monoamonium phosphate) & 0,25 & 1,09 & 1,92 & 2,76 & 3,59 & 4,43 \\
\hline Calcário calcítico (Limestone) & 1,53 & 1,53 & 1,53 & 1,53 & 1,53 & 1,53 \\
\hline DL-metionina (DL-methionine) & 0,08 & 0,08 & 0,08 & 0,08 & 0,08 & 0,08 \\
\hline Vitamina $\mathrm{C}^{3}$ (Vitamin $\left.C\right)$ & 0,05 & 0,05 & 0,05 & 0,05 & 0,05 & 0,05 \\
\hline Premix vitamínico e mineral ${ }^{4}$ (Vitamin and mineral mix) & 0,50 & 0,50 & 0,50 & 0,50 & 0,50 & 0,50 \\
\hline Sal (Salt) & 0,50 & 0,50 & 0,50 & 0,50 & 0,50 & 0,50 \\
\hline BHT (antioxidante) (Antioxidant) & 0,02 & 0,02 & 0,02 & 0,02 & 0,02 & 0,02 \\
\hline \multicolumn{7}{|l|}{$\begin{array}{l}\text { Composição calculada } \\
\text { Calculated composition }\end{array}$} \\
\hline $\mathrm{PB}(\%)(C P, \%)$ & 32,00 & 32,00 & 32,00 & 32,00 & 32,00 & 32,00 \\
\hline $\operatorname{ED}(\mathrm{kcal} / \mathrm{kg})^{2}(D E, \quad k c a l / k g)$ & 3.000 & 3.000 & 3.000 & 3.000 & 3.000 & 3.000 \\
\hline $\mathrm{FB}(\%)(C F, \%)$ & 4,45 & 4,45 & 4,45 & 4,45 & 4,45 & 4,45 \\
\hline $\mathrm{EE}(\%)$ & 6,68 & 6,68 & 6,68 & 6,68 & 6,68 & 6,68 \\
\hline $\mathrm{Ca}$ total $(\%)$ (Total $\mathrm{Ca}, \%)$ & 0,80 & 0,80 & 0,80 & 0,80 & 0,80 & 0,80 \\
\hline P total $(\%)^{1}$ (Total P, \%) & 0,55 & 0,73 & 0,94 & 1,14 & 1,37 & 1,59 \\
\hline Metionina + cistina digestível $(\%)^{2}$ (Digestible methionine + cystine, \%) & 0,95 & 0,95 & 0,95 & 0,95 & 0,95 & 0,95 \\
\hline Lisina digestível $(\%)^{2}$ (Digestible lysine, \%) & 1,72 & 1,72 & 1,72 & 1,72 & 1,72 & 1,72 \\
\hline Treonina digestível $(\%)^{2}$ (Digestible threonine, \%) & 1,13 & 1,13 & 1,13 & 1,13 & 1,13 & 1,13 \\
\hline Triptofano digestível $(\%)^{2}$ (Digestible tryptophan, \%) & 0,41 & 0,41 & 0,41 & 0,41 & 0,41 & 0,41 \\
\hline
\end{tabular}


oxigênio dissolvido na água foram aferidos a cada sete dias, respectivamente, utilizando-se potenciômetro e oxímetro. O fotoperíodo foi mantido em 12 horas de luz, por meio de iluminação proveniente de lâmpadas mistas, controlada por timer automático.

As dietas experimentais foram peletizadas e fornecidas em seis refeições diárias $(8,10,12,14,16$ e $18 \mathrm{~h})$ em pequenas quantidades para que possibilitasse a ingestão máxima sem que houvesse perdas. Foi realizada a limpeza diária dos aquários por meio de sifonagem, após a leitura matinal da temperatura da água, para retirada das fezes.

Foram avaliados o ganho de peso (GP), o consumo de ração (CR), a conversão alimentar (CA) e as taxas de crescimento específico (TCE), de eficiência de fósforo (TEFOS) e de eficiência protéica (TEP). Avaliaram-se ainda a composição químico-corporal (teores de umidade, proteína, gordura, cálcio, fósforo e relação corporal cálcio:fósforo) e a taxa de eficiência de retenção de fósforo no ganho de peso (TERF).

Para avaliação do desempenho, os peixes foram pesados, após jejum de 24 horas, no início e ao final do experimento. O GP foi obtido pela diferença entre os pesos médios final e inicial e a CA, pela diferença do consumo de ração pelo ganho de peso dos peixes.

A TCE foi determinada conforme a equação abaixo, utilizando-se transformações logarítmicas:

ln peso médio final $(\mathrm{g})$ - $\ln$ peso médio inicial $(\mathrm{g})$ $\mathrm{TCE}=$

tempo de experimento (dias)

A TEFOS e TEP foram obtidas por meio da divisão entre o ganho de peso médio dos peixes e o respectivo consumo de fósforo e proteína no período experimental.

Para as análises corporais, as tilápias foram sacrificadas em um isopor contendo gelo e congeladas no início (20\% da quantidade de peixes utilizados no experimento) e ao final do experimento (todos os peixes da unidade experimental). Estes peixes foram utilizados na determinação da TERF e da composição corporal.

A TERF foi obtida pela seguinte equação:

$$
\mathrm{TERF}=\frac{\left(\operatorname{Pfx} \% \mathrm{~F}_{\mathrm{f}}\right)-\left(\mathrm{Pix} \% \mathrm{~F}_{\mathrm{i}}\right)}{\mathrm{CR} \mathrm{x} \% \mathrm{~F}}
$$

em que: $\mathrm{P}_{\mathrm{f}}=$ peso médio final dos peixes; $\mathrm{F}_{\mathrm{f}}=$ porcentagem média de fósforo corporal final; $\mathrm{P}_{\mathrm{i}}=$ peso médio inicial dos peixes; $\mathrm{F}_{\mathrm{i}}=$ porcentagem média de fósforo corporal incial; $\mathrm{CR}=$ consumo de ração $; \mathrm{F}=$ porcentagem de fósforo na dieta experimental.
As análises bromatológicas das amostras dos peixes foram realizadas no Laboratório de Nutrição Animal do Departamento de Zootecnia (LNA/DZO) da Universidade Federal de Viçosa - UFV, conforme procedimentos descritos por Silva (1990).

As análises estatísticas foram realizadas utilizando-se o programa SAEG - Sistema de Análises Estatísticas e Genéticas. Os dados foram interpretados por meio de análises de variância e regressão (linear, quadrático ou descontínuo "Linear Response Plateau" (LRP)), a 5\% de probabilidade, conforme o melhor ajuste obtido para cada variável, com base na significância dos coeficientes de regressão pelo teste $\mathrm{F}$, no coeficiente de determinação, na soma de quadrado dos desvios e no fenômeno em estudo.

\section{Resultados e Discussão}

Foram obtidos valores de $28,0 \pm 0,66^{\circ} \mathrm{C}$ para temperatura da água, de $6,8 \pm 0,18$ para o $\mathrm{pH}$ e de $6,7 \pm 0,1 \mathrm{mg} / \mathrm{L}$ para o oxigênio dissolvido. Valores similares a estes foram citados por Popma \& Green (1990) e Kubitza (2000) como adequados para criação desta espécie.

Conforme demonstrado na Tabela 2, não foi observado efeito $(\mathrm{P}>0,05)$ dos níveis de fósforo da dieta sobre o GP dos animais. Este resultado foi semelhante ao encontrado por Asgard \& Shearer (1997) e Miranda et al. (2000), que, avaliando níveis de fósforo disponível em dietas para salmão e para tilápia-do-nilo, respectivamente, também não verificaram variação significativa no GP dos peixes entre os diferentes tratamentos.

De forma contrária, Satoh \& Borlongan (2001)e PimentelRodrigues \& Oliva-Teles (2001) observaram aumento linear no ganho de peso de juvenis de milkfish e gilthead sea bream, respectivamente, com a elevação dos níveis de fósforo da dieta.

Apesar de não ter variado significativamente, foi observada melhora gradativa de até $21,30 \%$ no GP dos animais entre os níveis de 0,55 a $1,14 \%$ de fósforo. A ausência de diferença significativa entre estes valores pode ser justificada pelo alto valor do coeficiente de variação obtido para esta variável.

Os níveis de fósforo das dietas não influenciaram $(\mathrm{P}>0,05)$ a taxa de crescimento específico (TCE). De forma semelhante, Haylor et al. (1988) e Oliva-Teles \& PimentelRodrigues (2004), estudando níveis de fósforo em dietas purificadas respectivamente, para alevinos de tilápia-donilo e juvenis de Europeu sea bass, não encontraram diferenças significativas para a TCE.

Em contrapartida, estes resultados diferem dos encontrados por Pimentel-Rodrigues \& Oliva-Teles (2001), em 
Tabela 2 - Ganho de peso (GP), taxa de crescimento específico (TCE), consumo de ação (CR) e conversão alimentar (CA) em função do nível de fósforo total da dieta

Table 2 - Weight gain (WG), specific growth rate (SGR), feed intake (FI) and feed/gain ratio (F/G) according to the dietary total phosphorus levels

\begin{tabular}{|c|c|c|c|c|c|c|c|}
\hline & \multicolumn{6}{|c|}{$\begin{array}{l}\text { Nível de fósforo total (\%) } \\
\text { Level of total phosphorus (\%) }\end{array}$} & \multirow[t]{2}{*}{$\mathrm{CV}(\%)^{1}$} \\
\hline & 0,55 & 0,73 & 0,94 & 1,14 & 1,37 & 1,59 & \\
\hline $\begin{array}{l}\text { PI (g) } \\
I W\end{array}$ & 0,63 & 0,61 & 0,62 & 0,62 & 0,62 & 0,61 & 10,38 \\
\hline $\begin{array}{l}\mathrm{PF}(\mathrm{g}) \\
F W\end{array}$ & 3,29 & 3,39 & 3,48 & 4,00 & 3,52 & 3,48 & 21,30 \\
\hline $\begin{array}{l}\text { GP (g) } \\
W G\end{array}$ & 2,66 & 2,78 & 2,86 & 3,38 & 2,90 & 2,87 & 20,21 \\
\hline $\begin{array}{l}\text { TCE } \\
S G R\end{array}$ & 4,16 & 4,29 & 4,32 & 4,59 & 4,32 & 4,36 & 8,95 \\
\hline $\begin{array}{l}\mathrm{CR}(\mathrm{g}) \\
F I\end{array}$ & 5,47 & 5,53 & 5,38 & 6,30 & 5,57 & 5,82 & 16,82 \\
\hline $\begin{array}{l}\mathrm{CA}^{2} \\
F / G \\
\end{array}$ & 2,05 & 1,99 & 1,90 & 1,90 & 1,93 & 2,03 & 5,96 \\
\hline
\end{tabular}

juvenis gilthead sea bream, e por Roy \& Lall (2003), em haddock, que observaram efeito significativo do nível de fósforo da dieta sobre a TCE dos animais.

As médias dos valores da TCE obtidos neste trabalho foram superiores às obtidas por Haylor et al. (1988), que utilizaram dietas purificadas para alevinos de tilápia-do-nilo. A utilização de dietas purificadas, que normalmente apresentam baixa palatabilidade, pode proporcionar taxas de crescimento inferiores às usualmente obtidas com dietas práticas, podendo, desta forma, influenciar nos resultados obtidos.

Não houve variação $(\mathrm{P}>0,05)$ no consumo de ração $(\mathrm{CR})$ à medida que se elevou o nível de fósforo das dietas. Os níveis de fósforo na dieta influenciaram a conversão alimentar aparente, que melhorou até o nível estimado de 1,10\% de fósforo (Figura 1).

O nível de $1,10 \%$ de $\mathrm{P}$ que proporcionou o melhor resultado de CA neste estudo ficou acima dos valores de 0,90\%, encontrado por Watanabe et al. (1980); e 0,80\%, obtido por Signor et al. (2004) em tilápias-do-nilo, utilizando dietas práticas. Foi superior ainda ao de $0,50 \%$ encontrado por Robinson et al. (1987) e Haylor et al. (1988) utilizando dietas purificadas.

Os resultados médios da taxa de eficiência de retenção fósforo (TERFOS), da taxa de eficiência de fósforo (TEFOS) e da taxa de eficiência protéica (TEP) são apresentados na Tabela 3.

A TERFOS e a TEFOS diminuíram de forma linear $(\mathrm{P}<0,05)$ à medida que se elevaram os níveis de fósforo na dieta, segundo as equações, respectivamente, $\hat{\mathrm{Y}}=28,6906$

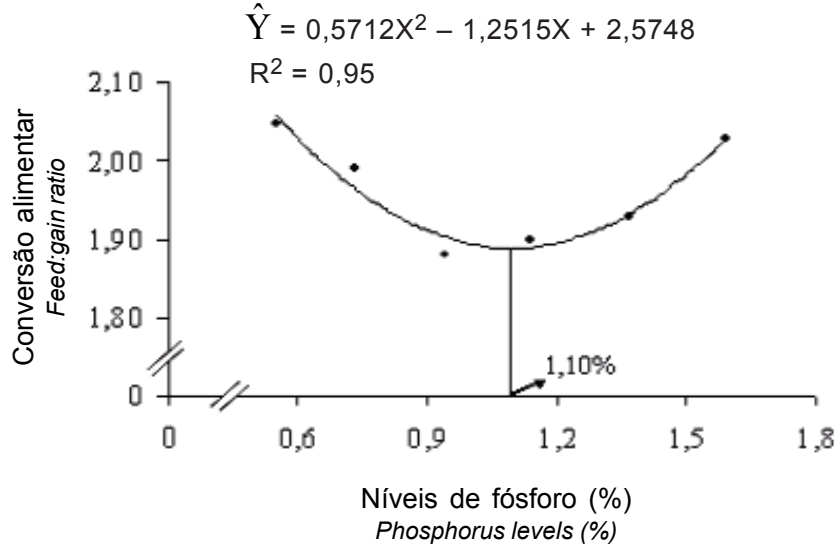

Figura 1 - Conversão alimentar em função do nível de fósforo da diet.

Figure 1 - Feed/gain ratio according to the dietary total phosphorus levels.

Tabela 3 - Taxa de eficiência de retenção de fósforo (TERFOS), taxa de eficiência de fósforo (TEFOS) e taxa de eficiência de protéica (TEP), em função do nível de fósforo total da dieta

Table 3 - Retention efficiency of rate of phosphorus(PRER), phosphorus efficiency rate (PER) and protein efficiency rate (PER) according to the dietary total phosphorus levels

\begin{tabular}{lllllll}
\hline & \multicolumn{4}{c}{$\begin{array}{c}\text { Nível de fósforo total (\%) } \\
\text { Total phosphorus level (\%) }\end{array}$} & CV (\%) ${ }^{1}$ \\
\cline { 2 - 7 } & 0,55 & 0,73 & 0,94 & 1,14 & 1,37 & 1,59 \\
\hline
\end{tabular}

$\begin{array}{llllllll}\text { TERFOS }(\%)^{1} & 26,06 & 22,52 & 17,24 & 15,14 & 11,87 & 9,85 & 14,80\end{array}$

PRER

TEFOS $(\mathrm{g} / \mathrm{g})^{2} \quad 91,95 \quad 69,85 \quad 58,08 \quad 47,71 \quad 38,74 \quad 31,78 \quad 5,80$

PER

TEP $(\mathrm{g} / \mathrm{g})^{3} \quad 1,52 \quad 1,57 \quad 1,66 \quad 1,66 \quad 1,62 \quad 1,54 \quad 6,25$

PER

CV - coeficiente de variação (coefficient of variation).

${ }^{1}$ Efeito linear (Linear effect): $(P<0,05) \hat{Y}=28,6906-3,0060 X$.

2 Efeito linear (Linear effect): $(P<0,05) \hat{Y}=123,4800-7,8813 X$.

${ }^{3}$ Efeito quadrático (Quadractic effect): $(P<0,05) \hat{Y}=-0,509 X^{2}+1,1162 X+$ 1,0507

$-3,0060 \mathrm{X} \mathrm{r}^{2}=0,97$ e $\hat{\mathrm{Y}}=123,4800-7,8813 \mathrm{Xr}^{2}=0,94 . \mathrm{Com}$ este resultado, ficou evidenciada a diminuição da eficiência de retenção corporal e da eficiência deste mineral para o ganho de peso com o aumento da quantidade de fósforo consumida.

Este resultado é semelhante ao encontrado por Miranda et al. (2000), que, estudando níveis de fósforo para tilápiado-nilo, observaram que a retenção de $\mathrm{P}$ diminuiu à medida que se elevou o nível de fósforo da dieta. Porém, resultado diferente foi encontrado por Haylor et al. (1988), que verificaram aumento na retenção de $\mathrm{P}$ em tilápias-do-nilo com o aumento do conteúdo desse mineral na ração, independentemente do nível de Ca. Entretanto, Nakamura (1982) observou relação linear positiva entre retenção de fósforo 


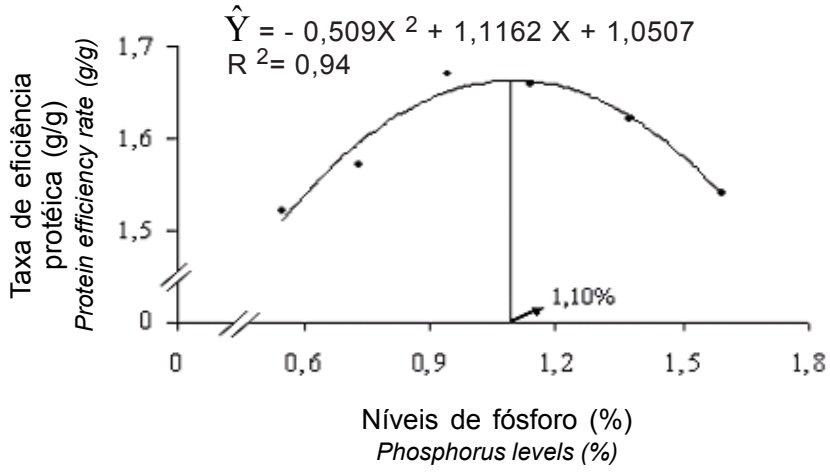

Figura 2 - Taxa de eficiência protéica em função do nível de fósforo da dieta.

Figure 2 - Protein efficiency rate according to the dietary total phosphorus levels.

em carpas com o aumento do conteúdo de fósforo da ração quando a concentração de cálcio foi de $0,70 \%$. Todavia, essa relação diminuiu quando a concentração de $\mathrm{Ca}$ foi de $1,00 \%$. Portanto, o teor de $\mathrm{Ca}$ da dieta pode afetar a taxa de eficiência de retenção de $\mathrm{P}$, sugerindo que níveis elevados de Ca na ração podem promover efeito inibitório sobre a absorção e/ou retenção de P.

Os níveis de $\mathrm{P}$ da dieta tiveram efeito $(\mathrm{P}<0,05)$ sobre a TEP, que melhorou até o nível estimado de $1,10 \%$ de fósforo (Figura 2).

O nível de $\mathrm{P}$ que resultou em melhor TEP neste estudo ficou acima dos níveis de 0,65 e $0,75 \%$ obtidos, respectivamente, por Oliva-Teles \& Pimentel-Rodrigues (2004), em European sea bass, e por Pimentel-Rodrigues \& Oliva-Teles (2001), em gilthead sea bream.

Os valores médios da composição corporal dos peixes são apresentados na Tabela 4. Para os teores corporais de umidade, proteína, gordura, fósforo, cálcio e a relação Ca:P, não foram observadas diferenças significativas $(\mathrm{P}<0,05)$ à medida que se elevou o nível de fósforo das dietas.

Corroborando esse resultado, Pimentel-Rodrigues \& Oliva-Teles (2001) não encontraram diferença significativa no conteúdo de umidade, proteína, fósforo, cálcio e gordura da carcaça de juvenis de gilthead sea bass. Oliva-Teles \& Pimentel-Rodrigues (2004), em juvenil Europeu sea bass, e Coloso et al. (2003), em truta arco-íris alimentados com dietas com diferentes níveis de $\mathrm{P}$, também não encontraram diferenças significativas na composição corporal da carcaça.

Em outros estudos, entretanto, correlação positiva entre o conteúdo de fósforo da carcaça e o nível de fósforo da dieta foi obtida (Ogino \& Takeda, 1978; Vielma \& Lall, 1998; Borlongan \& Satoh, 2001), sendo que o conteúdo de $\mathrm{P}$ corporal foi significativamente menor quando os peixes foram alimentados com dieta deficiente em $P$ por um longo período experimental.

Alguns autores observaram que o aumento no teor de $\mathrm{P}$ da dieta resultou em redução do conteúdo de gordura da carcaça e aumento do teor de umidade (Takeuchi \& Nakazoe, 1981; Vielma et al., 2002; Roy \& Lall, 2003). Esses autores especularam que a deficiência de $\mathrm{P}$ na dieta pode provocar a inibição da ß-oxidação dos ácidos graxos, o que aumenta a acumulação de gordura corporal.

As pesquisas realizadas para determinação dos valores de exigência de P para tilápia-do-nilo são muito discrepantes, com valores de 0,50 a 0,90\% (Watanabe et al., 1980; Viola et al., 1986; Robinson et al., 1987; Haylor et al., 1988; NRC, 1993; Signor et al., 2004). Essa variação pode estar relacionada à fase de desenvolvimento dos peixes utilizados nos experimentos, visto que as exigências dos animais podem variar nos diferentes estádios de vida.

Outros fatores, como temperatura da água, linhagem dos animais utilizados nos experimentos e critério de resposta utilizado para definir o nível de fósforo da dieta, podem influenciar na determinação da exigência. Além disso, o modelo estatístico adotado também pode proporcionar variação entre os valores de exigência de fósforo obtidos, sendo que na maior parte das pesquisas, como as conduzidas por Haylor et al. (1988) e Signor et al. (2004), foram utilizados testes de média para determinar a variação obtida nas variáveis avaliadas.

Tabela 4 - Composição corporal em função do nível de fósforo total da dieta

Table 4 - Body composition according to the dietary total phosphorus levels

\begin{tabular}{|c|c|c|c|c|c|c|c|c|}
\hline & \multicolumn{6}{|c|}{$\begin{array}{l}\text { Nível de fósforo total (\%) } \\
\text { Total phosphorus level (\%) }\end{array}$} & \multirow[t]{2}{*}{$\mathrm{CV}(\%)$} & \multirow[t]{2}{*}{$\mathrm{CPI}^{1}$} \\
\hline & 0,55 & 0,73 & 0,94 & 1,14 & 1,37 & 1,59 & & \\
\hline Umidade (\%) (Humidity) & 72,64 & 73,81 & 72,94 & 73,71 & 73,97 & 73,14 & 8,11 & 80,01 \\
\hline Proteína $(\%)$ (Protein) & 12,37 & 12,56 & 12,63 & 12,54 & 12,87 & 12,34 & 7,79 & 11,42 \\
\hline Gordura (\%) (Fat) & 11,50 & 9,89 & 11,19 & 10,48 & 9,96 & 11,02 & 10,26 & 4,12 \\
\hline Fósforo (\%) (Phosphorus) & 0,32 & 0,35 & 0,33 & 0,34 & 0,34 & 0,34 & 9,56 & 0,48 \\
\hline Cálcio (\%) (Calcium) & 0,56 & 0,59 & 0,59 & 0,61 & 0,61 & 0,60 & 11,20 & 0,82 \\
\hline Rel. $\mathrm{Ca} / \mathrm{Ps}$ (Ca/P ratio) & 1,74 & 1,69 & 1,78 & 1,80 & 1,80 & 1,78 & 8,03 & 1,71 \\
\hline
\end{tabular}

CV - coeficiente de variação (CV - coefficient of variation).

${ }^{1}$ Composição corporal inicial (Initial body composition). 


\section{Conclusões}

A exigência de fósforo total para alevinos de tilápia-donilo é de $1,10 \%$ por proporcionar a melhor resposta na conversão alimentar aparente e na taxa de eficiência protéica.

\section{Literatura Citada}

ASGARD, T.; SHEARER, K.D. Dietary phosphorus requirement of juvenile Atlantic salmon, Salmo salar L. Aquaculture Nutrition, v.3, p.17-23, 1997.

BORLONGAN, I.J.; SATOH, S. Dietary phosphorus requirement of juvenile milkfish, Chanos chanos (Forsskal). Aquaculture Research, v.32, p.26-32, 2001.

COLOSO, R.M.; KING, K.; FIETCHER, J.W. et al. Phosphorus utilization in rainbow trout (Oncorhynchus mykiss) fed pratical diets and its consequences on effluent phosphorus levels. Aquaculture, v.144, p.227-237, 2003.

ENGLISH, W.R.; SCHWEDLER, T.E.; DYCK, L.A. Aphanizomenon flos-quae, a toxic blue green alga in commercial channel catfish, Ictalurus punctatus, ponds: a case history. Journal of Applied Aquaculture, v.3, p.195-209, 1993.

FURUYA, W.M.; PEZZATO, L.E.; PEZZATO, A.C. et al. Coeficientes de digestibilidade e valores de aminoácidos digestíveis de alguns ingredientes para tilápia-do-nilo (Oreochromis niloticus). Revista Brasileira de Zootecnia, v.30, n.4, p.1143-1149, 2001.

HAYLOR, G.S.; BEVERIGDE, M.C.M.; JAUNCEY, K. Phosphorus nutrition of juvenile Oreochromis niloticus. In: THE SECOND INTERNATIONAL SYMPOSIUM ON TILAPIA IN AQUACUlture, 1998, Manila. Proceedings... Manila: Department of Fisheries, 1988. p.341-345.

HILDSORF, A.W.S. Genética e cultivo de tilápias vermelhas, uma revisão. Boletim do Instituto de Pesca, v.22, p.73-87, 1995.

KUBITZA, F. Tilápia: tecnologia e produção comercial. Jundiaí: Divisão de Biblioteca e Documentação da USP, 2000. 285p.

MIRANDA, E.C.; PEZZATO, A.C.; PEZZATO, L.E. et al. Relação cálcio/fósforo disponível em rações para tilápia-do-nilo (Oreochromis niloticus). Revista Brasileira de Zootecnia, v.29, n.6, p.2162-2171, 2000.

NAKAMURA, Y. Effects of dietary phosphorus and calcium contents on the absorption of phosphorus in the digestive tract of carp. Bulletin of the Japanese Society of Scientific Fisheries, v.48, p.409-13, 1982.

NATIONAL RESEARCH COUNCIL - NRC. Nutritional requirements of fishes. Washington, D.C.: Academic Press, 1993. 114p.

OGINO, C.; TAKEDA H. Requirements of rainbow trout for dietary calcium and phosphorus. Bulletin of the Japanese Society of Scientific Fisheries, v.44, p.1019-1022, 1978.

OLIVA-TELES, A.M.; PIMENTEL-RODRIGUES, A. Phosphorus requirement of sea bass. Aquaculture Research, v.35, p.636$642,2004$.

PIMENTEL-RODRIGUES, A.; OLIVA-TELES, A.M. Phosphorus requirement of gilthead sea bream (Sparus aurata L.). Aquaculture Research, v.32, p.157-161, 2001.

POPMA, T.J.; GREN, B.W. Sex reversal of tilapia in earthen ponds. Aquaculture production manual. Alabama: Auburn University, Alabama Research and Development, 1990. 15p (Series 35).
ROBINSON, E.H.; LABOMASCUS, D.; BROWN, P.B. et al. Dietary calcium and phosphorus requirements of Oreochromis aureus reared in calcium- free water. Aquaculture, v.64, p.267-279, 1987.

ROY, P.K.; LALL, S.P. Dietary phosphorus requirements of juvenile haddock (Melanogrammus aeglefinus L.). Aquaculture, v.221, p.451-468, 2003.

SATOH, S.; BORLONGAN, I.G. Dietary phosphorus requirements of juvenile milkfish, Chanos chanos (Forsskal). Aquaculture Research, v.32, p.26-32, 2001.

SIGNOR, A.; BOSCOLO, W.R.; FEIDEN, A. et al. Exigência de fósforo para alevinos de tilápia-do-nilo.In: REUNIÃO ANUAL DA SOCIEDADE BRASILEIRA DE ZOOTECNIA, 41., 2004, Campo Grande. Anais... Campo Grande: Sociedade Brasileira de Zootecnia, 2004. p.441.

SILVA, D.J. Análise de alimentos (métodos químicos e biológicos). 2.ed. Viçosa: Universidade Federal de Viçosa, 1990. 165p.

SIMKISS, K. Calcium metabolism of fish in relation to ageing. In: BAGEnAL, T.B. (Ed.) The ageing of fish. Surrey: Unwin Brothers Ltda, 1974. 234p.

TAKEUCHI, M.; NAKAZOE, J. Effect of dietary phosphorus on lipid content and its composition in carp. Bulletin of the Japanese Society of Scientific Fisheries, v.47, p.347-52, 1981.

Van der PLOEG, M.; BOYD, C.E. Geosmin production by cyanobacteria (blue green algae) in fish ponds at Auburn, Alabama. Journal World Aquaculture Society, v.22, p.207216, 1991.

Van der PLOEG, M.; TUCKER, C.S. Seasonal trends in flavor quality of channel catfish, Ictalurus punctatus, from commercial ponds in Mississippi. Journal of Applied Aquaculture, v.3, p.121$140,1994$.

VIELMA, J.; LALL, S.P. Phosphorus utilization by Atlantic salmon (Salmo sala) reared in freshwater is not influenced by higher dietary calcium intake. Aquaculture, v.160, p.117-128, 1998.

VIELMA, J.; KOSKEIA J.; RUOHONEN K. Growth, bone mineralization, and heat and low oxygen tolerance in Europan whitefish (Coregonus lavaretus L.) fed with graded levels of phosphorus. Aquaculture, v.212, p.321-333, 2002.

VIOLA, S.; ZOHAR. G.; ARIELE, Y. Requirements of phosphorus and its availability from differents sources for intensive pond culture species in Israel. Part II - carp culture. Bamidgeh, v.38, p.44-54, 1986.

WATANABE, T.; TAKEUCHI, T.; MURUKAMI, A. et al. The availability to Oreochromis niloticus of phosphorus in white fish meal. Bulletin of the Japanese Society of Scientific Fisheries, v.46, p.897-900, 1980 .

WATANABE, T. Fish nutrition and maniculture. Kanagawa: Japan Cooperation Agency Textbook; Kanagawa International Training Center, 1988. 233p.

WILSON, R.P.; ROBINSON E.H.; GATLIN, D.M.III et al. Dietary phosphorus requirement of channel catfish. Journal Nutrition, v.112, p.1197-1292, 1982.
Recebido: 07/11/05 Aprovado: 10/04/06 